UVX 2010 (2011) 63-67

DOI: $10.1051 / \mathrm{uvx} / 2011009$

(C) Owned by the authors, published by EDP Sciences, 2011

\title{
The European XFEL in Hamburg: Status and beamlines design
}

\author{
J. Gaudin, H. Sinn and Th. Tschentscher \\ European XFEL GmbH, Albert-Einstein-Ring 19, 22761 Hamburg, Germany
}

\begin{abstract}
The European XFEL is a hard x-ray Free-Electron Laser currently under construction in Hamburg. This international facility will deliver in 2015 femtosecond pulses of soft to hard x-ray light. In this article, we present the main characteristics of the facility and give a brief overview of the foreseen science to be adressed. An emphasize will be given on the beamline design. In fact x-ray FEL beam present specific and completely new characteristics which require the development of new technical solution. The on-going effort to tackle all the relevant challenges is presented, as well an overview of the conceptual design for the different European XFEL beamlines.
\end{abstract}

\section{INTRODUCTION}

Linear accelerator based lasers, so called Free Electron Lasers (FEL) are now becoming a reality as 4th generation x-ray light sources. Based on the SASE (Self Amplified Spontaneous Emission) process, they deliver ultra-short coherent light in the $\mathrm{x}$-ray photon energy range (see references [1,2] for a comprehensive review). After the first successful development of FEL in the soft x-ray region at FLASH (Free-electron LASer in Hamburg), several x-ray facilities are under construction (European XFEL in Hamburg, XFEL/SPRING8 in Japan) or recently started (LCLS in Stanford).

In the first part of this article, we will give a brief overview of the European XFEL which is under construction in Hamburg since the beginning of 2009. We refer to the technical design for a full and detailed description. Starting from 2015, this facility will deliver ultra-short pulses of soft to hard $\mathrm{x}$-rays. The scientific experiments will be performed at six different experimental stations, each devoted to a specific field ranging from the study of high energy density matter to single bio-molecule imaging. In the second part, the beamlines design dealing with the intense, coherent and highly collimated FEL beam is outlined. The resulting conceptual design of the beamlines is then finally presented.

\section{THE EUROPEAN XFEL: MAIN CHARACTERISTICS}

The European XFEL will come on duty in the early 2015. The operation can be briefly described in the following manner: Electrons generated from a solid cathode by an optical laser beam are accelerated by a radio frequency gun up to $120 \mathrm{MeV}$. The electron bunches are then steered to the linear accelerator (linac). While passing through the $1.6 \mathrm{~km}$ long sequence of superconductor modules the electrons are accelerated up to $17.5 \mathrm{GeV}$. The typical bunch charge will be up to $1 \mathrm{nC}$ with a peak current close to $\mathrm{I}=5 \mathrm{kA}$. The use of superconducting cavities allows high repetition rate operation modes. The machine will hence deliver 27000 pulses / second distributed in bunch trains containing each 2700 pulses at $4.5 \mathrm{MHz}$ repetition rate (i.e. separated by $220 \mathrm{~ns}$ ). The $600 \mu$ s long bunch trains have then a $10 \mathrm{~Hz}$ repetition rate.

Once accelerated at the proper energy the electron bunches are distributed to two beamlines including three FEL undulators sections. The first electron beamline leads to the SASE1 and SASE3

This is an Open Access article distributed under the terms of the Creative Commons Attribution-Noncommercial License 3.0, which permits unrestricted use, distribution, and reproduction in any noncommercial medium, provided the original work is properly cited. 
Table 1. Summary of the photon beamlines and scientific instruments. FEL parameters are given for a $1 \mathrm{nC}$ bunch charge [3]

\begin{tabular}{|c|c|c|l|}
\hline & $\begin{array}{c}\text { Wavelength } \\
\text { Pulse duration } \\
\text { Photons / pulse }\end{array}$ & instrument & \\
\hline \multirow{3}{*}{ SASE 1 } & $0.1 \mathrm{~nm}$ & SPB & technique \\
\cline { 3 - 4 } & $100 \mathrm{fs}$ & MID & $\begin{array}{l}\text { Coherent diffraction imaging by single particles } \\
\text { X-ray photon correlation spectroscopy }\end{array}$ \\
\hline \multirow{3}{*}{ SASE 2 } & $0.1-0.4 \mathrm{~nm}$ & FXE & $\begin{array}{l}\text { Time resolved diffraction scattering } \\
\text { Time resolved spectroscopy }\end{array}$ \\
\cline { 3 - 4 } & $100 \mathrm{fs}$ & & X-ray generation/probing of extremes states of matter \\
\cline { 3 - 4 } SASE 3 & $1-16 \times 10^{12}$ & HED & Electron and ion spectroscopy \\
\cline { 3 - 4 } & $100 \mathrm{fs}$ & SQS & Coherent diffraction imaging \\
& $1.2-3310^{13}$ & SCS & $\begin{array}{l}\text { X-ray photon correlation spectroscopy } \\
\text { Photon-in and photon-out spectroscopy }\end{array}$ \\
\cline { 3 - 4 } & & &
\end{tabular}

undulators, while the second one leads to the SASE2 undulator. Each of these undulators sections produces x-ray radiation by the SASE process. The generated x-rays are delivered to six different experimental stations dedicated to a specific field of research. The main parameters of the x-ray pulses together with brief description of the experimental stations are summarized in table 1.

\section{BEAMLINES DESIGN}

\subsection{FEL radiation properties}

The main characteristics of an FEL beam are the high photon flux, the coherence properties and the femtosecond pulse duration. In order to be able to employ these unique features the beamlines should be designed in such a way that they enable transport of the FEL beam with minimum degradation of quality. The main goal of design is constrained by the following requirements:

- fulfil the requirements of the users

- preserving the beam quality (flux, coherence, pulse duration)

- ensure a flexible and safe operation mode

For each experimental station the parameters: photon energy range, pulse energy, radiation bandwidth... have been determined following the requirements of the user community define at the occasion of different specific workshops [4].

The second point is by far the most difficult one. A complete program of fundamental research has then been set-up at the European XFEL in order to tackle all the relevant challenges. If the coherence depends only on the source parameters, the experiments which rely on this property also require a high wavefront quality. The wavefront is influenced along the transport by the optical system. For short wavelengths, in the sub nm range, very strict requirements concerning the mirror length and surface quality follow from simulation of wavefront propagation. For a $0.1 \mathrm{~nm}$ wavelength an rms surface error height smaller than $2 \mathrm{~nm}$ [5] over the entire length of the mirror is required.

The preservation of the flux leads to another challenge which is the safe operation of the x-ray optics. For a few $\mathrm{mJ}$ per pulse, the fluence at the surface of the mirrors for example, can be high enough to reach the damage threshold. Different experiments have been carried out in order to determine the damage threshold of materials $[6,7]$ and in the specific x-ray mirror geometry $[8,9]$. Concerning the mirrors coating, low $\mathrm{Z}$ material are preferred in order to achieve good reflectivity and high radiation hardness. The existing facilities use amorphous-Carbon or $\mathrm{B}_{4} \mathrm{C}$. In the case of the European XFEL amorphous-Carbon is foreseen. 
Another challenge is the heat load due the unique high repetition rate of the European XFEL. In fact, the $600 \mu$ s train containing 2700 pulses with more than $1 \mathrm{~mJ}$ per pulses lead to an average load on optical elements that can exceed $10 \mathrm{~kW} / \mathrm{cm}^{2}[10]$. The heat will be even higher for the $1^{\text {st }}$ mirror, which is also irradiated by hard X-ray spontaneous emission emitted by the FEL undulator. Deformation of the mirrors has to be limited to a very small level to preserve good quality beam. The conceptual design therefore foresees to remove the heat load a highly efficient cooling scheme. The mirror substrates for will be $\mathrm{Si}$, and cooling schemes are under development.

\subsection{Hard $x$-ray beamlines: SASE 1 and SASE 2}

The SASE 1 and SASE 2 beamlines will deliver photons in the range of 0.1 to $0.4 \mathrm{~nm}$ but extension to shorter wavelength seems possible. The conceptual design for these beamlines [10] is shown in figure 1. They differ only in the distances from the undulator end. The first optical elements are a pair of plan mirrors used to shift the beam by $50 \mathrm{~mm}$ optical axis. This system aims at blocking the direct line of sight from the undulator to the experimental hall. The angle of incidence of the $1^{\text {st }}$ mirror will be tuneable in order to accommodate $4 \sigma$ to $6 \sigma$ ( $\sigma$ is defined in the case of a Gaussian profile as the equivalent of the root mean square) of the projected beam on a $800 \mathrm{~mm}$ long mirror.

The $3^{\text {rd }}$ distribution mirror (in fact 2 mirrors) allows steering the beam to three different locations separated at the far end by a $1.45 \mathrm{~m}$ horizontally. Two experimental stations will be located at each line. As the incident angle of these distribution mirrors can not be tuned the size of the beam has to be reduced at this location. This is achieved by the $2^{\text {nd }}$ offset mirror which will be bendable. A typical radius of $20 \mathrm{~km}$ will allow fulfilling the $4 \sigma$ to $6 \sigma$ condition on the distribution mirrors. The beam can then propagate freely until the experimental hall. Strong focussing optics (not shown in the figure 1) will be placed close to the sample position inside the experimental hall. Large aperture zone plates based optics as well as bendable mirrors are foreseen for this purpose.

The figure 1 also shows crystal based monochromators: a double crystal diamond and a double crystal silicon are currently under development. The achievable resolving power should be in the order of $10^{4}$ or better. Finally the beamline is complemented by beam position and energy monitors, high power slits in order to cut the background radiation, and solids attenuators for tuning the energy of the pulse.

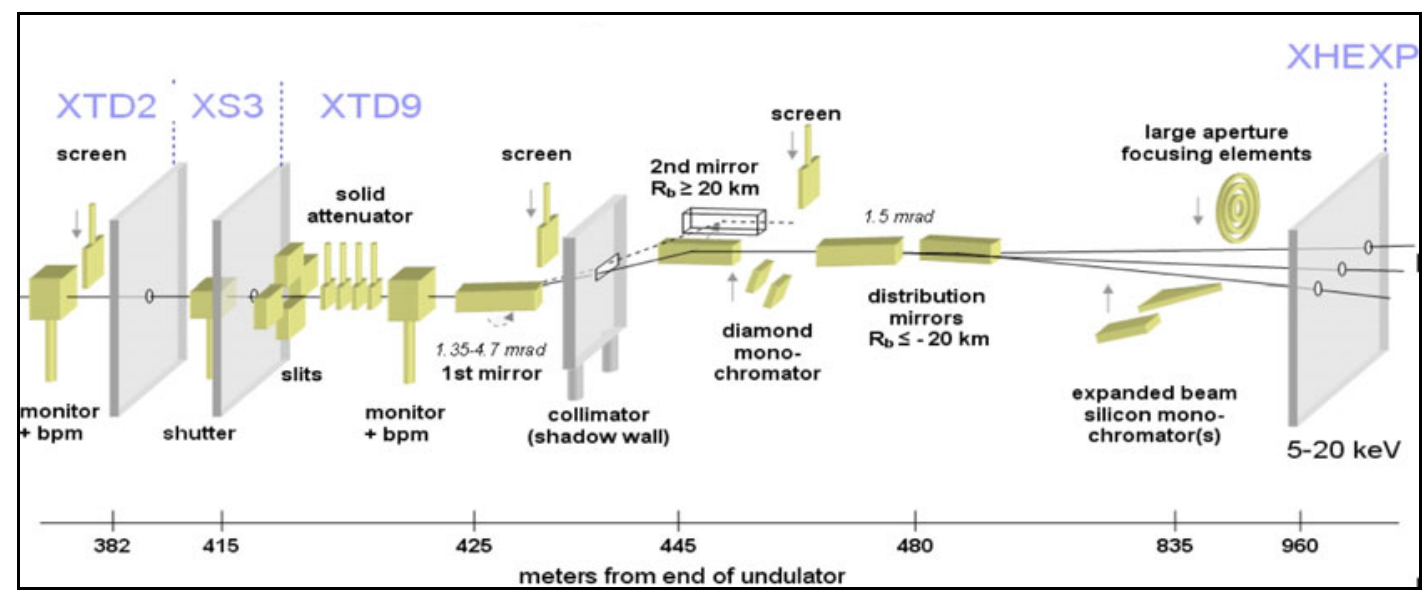

Figure 1. Schematic diagram of the SASE 1 and SASE 2 beamlines. 


\subsection{Soft x-ray bameline: SASE 3}

The SASE 3 beamline will be dedicated to soft x-ray range between 0.4 and $3 \mathrm{~nm}$. The SASE 3 beamline will comprise 2 branches, as shown in figure 2, one "pink beam" dedicated to the study of non linear processes and a monochromatic beam for spectroscopic applications. As for the SASE 1 and SASE 2 , the $1^{\text {st }}$ optical elements are the offset mirrors. The angle of the $1^{\text {st }}$ mirror will be tuneable in order to accommodate $4 \sigma$. For the longer wavelengths of SASE 3 the $4 \sigma$ condition seems to be sufficient to preserve good wavefront quality. The $2^{\text {nd }}$ mirror will be translated in order to keep the optical axis unchanged. In the pink beam configuration the beam will propagates freely until the experimental hall, where focussing will be achieved by a pair of bendable mirrors in the KB (Kirkpatrick Baez [11]) configuration.

In the monochromatic configuration a $3^{\text {rd }}$ mirror will be used to steer the beam to the monochromator. The final design is underway but presents several challenges. The scheme foreseen is based on a plane grating monochromator (PGM) [12]. As long grating are presently not available the mirror M3 will also be used to focus in the horizontal direction the beam. The projected foot print in the horizontal plane needs to be reduced. The design aims at achieving a resolving power of $10^{4}$ over a broad energy range. After the exit slit (not shown in the figure 1), the beam propagates to the experimental hall where focussing optics will be set-up. The basic focussing configuration would be KB mirrors with focal spot in the $1 \mu \mathrm{m}$ range.

As in the case of SASE1 and 2, the beamline is completed with beam position monitors (BPM in figure 2) as well as energy monitor similar to what has been developed for FLASH in the VUV range [13]. Finally a $40 \mathrm{~m}$ long gas attenuator will allow to continuously tuning the energy per pulse [14].

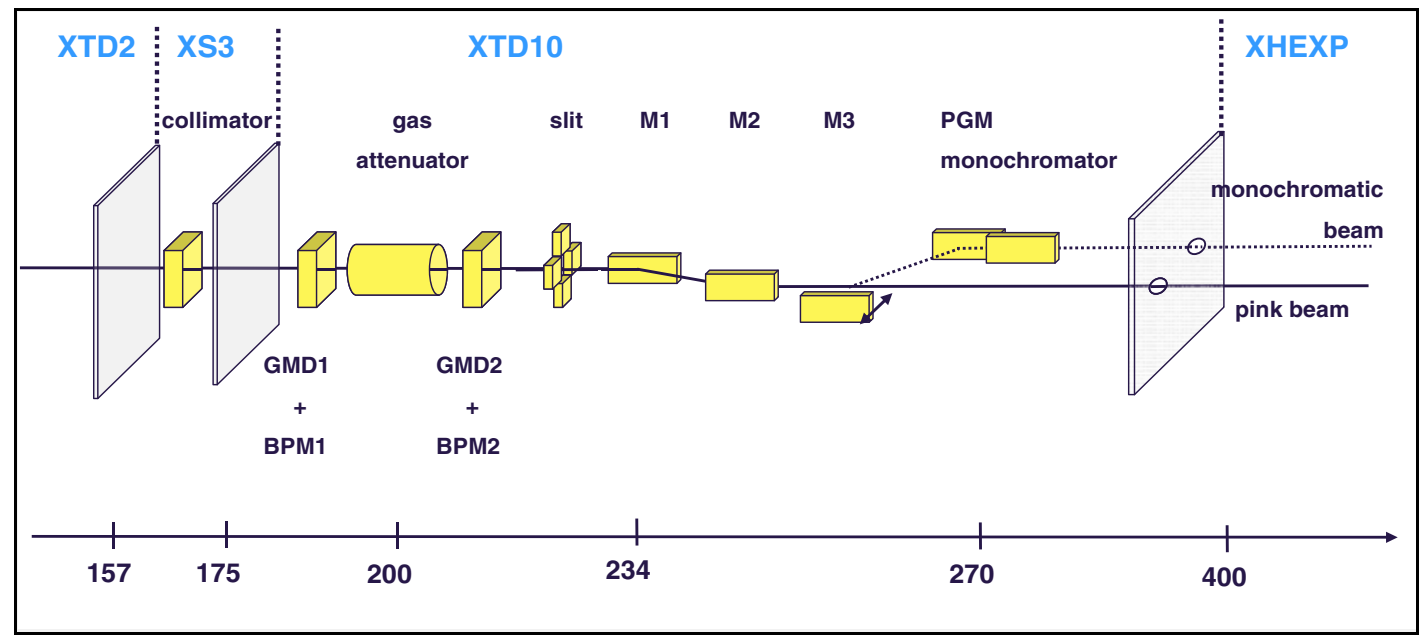

Figure 2. Schematic diagram of the SASE 3 beamline.

\section{CONCLUSION}

The main characteristics of the European XFEL were presented as well as considerations for the conceptual design of the beamlines. The principle of the design is to preserve the unique properties of the FEL beam in terms of coherence, photon flux and temporal resolution required to perform the most demanding experiments. A large effort ranging from engineering studies to fundamental research is currently going on. 


\section{References}

[1] Saldin E.L. et al., Physics Reports 260 (1995) 187

[2] Kahn S., Journal of Modern Optics 55 (2008) 3469

[3] Altarelli M. et al., The European X-ray free-electron laser Technical design report (M. Altarelli, Hamburg, 2006)

[4] Detailed reports of these workshops are available on the European XFEL website: www.xfel.eu

[5] Geloni G. et al. , New. J. Phys. 12 (2010) 035021

[6] Levy et al. Appl. Phys. Lett. 96 (2010) 151114

[7] Hau-Riege S. et al., Opt. Expr. (2010) in press

[8] Chalupsky et al. Appl. Phys. Lett. 95 (2009) 031111

[9] Dastjani S. et al., Nucl. Instrum. and Methods A (2010) in press

[10] Sinn H. et al., "Design of photon beamlines at the European XFEL", $32^{\text {nd }}$ International FEL Conference, Malmö August 2010, in press

[11] Kirkpatrick P. and Baez A.V., J. Opt. Soc. Am 38 (1948) 776

[12] Follath, R. and Senf F., Nucl. Instrum. and Methods A 390 (1997) 388

[13] Tiedtke et al., New J. Phys. 11 (2009) 023029

[14] Hahn U. and Tiedtke K. "The Gas attenuator of FLASH at DESY", Synchrotron Radiation Instrumentation: Ninth International Conference, J-Y. Choi and S. Rah Eds. (AIP, 2007) pp 276-279 